\title{
On Start Point Selection for Time-Optimal System Design Algorithm
}

\author{
ALEXANDER ZEMLIAK, PEDRO MIRANDA \\ Department of Physics and Mathematics \\ Puebla Autonomous University \\ Av. San Claudio s/n, Puebla, 72570 \\ MEXICO
}

\begin{abstract}
On the basis of generalized theory of system design the behavior of the different design trajectories in the design phase space was analyzed. The problem of the initial point selection for the time-optimal algorithm construction was connected with the discovering of an additional acceleration effect of design process. This acceleration effect has been discovered by the analysis of various design strategies with different initial points. This effect can be understood well on the basis of the elaborated design methodology by means of the different design trajectory analysis. It is displayed for all analyzed circuits and it reduces additionally the total computer time for the system design. Numerical results of some passive and active nonlinear electronic circuit design demonstrate the perspective of the proposed approach.
\end{abstract}

Key-Words: - Time-optimal design algorithm, control theory formulation, acceleration effect.

\section{Introduction}

The generalized theory for the system design on the basis of control theory formulation was elaborated in some previous works [1]-[3]. This approach serves for the time-optimal design algorithm definition. On the other hand this approach gives the possibility to analyze with a great clearness the design process while moving along the trajectory curve into the design space. The main conception of the theory is the introduction of the special control functions, which, on the one hand generalize the design process and, on the other hand, they give the possibility to control design process to achieve the optimum of the design objective function for the minimum computer time. This possibility appears because practically an infinite number of the different design strategies that exist within the bounds of the theory, but the different design strategies have the different operation number and executed computer time. On the bounds of this conception, the traditional design strategy is only a one representative of the enormous set of different design strategies. As shown in [3] the potential computer time gain that can be obtained by the new design problem formulation increases when the size and complexity of the system increase but it is realized only in case when we have the algorithm for the optimal trajectories real construction. We can define the formulation of the intrinsic properties and special restrictions of the optimal design trajectory as one of the first problems that needs to be solved for the optimal algorithm construction.

\section{Acceleration effect}

On the basis of the new design methodology an additional acceleration effect of the design process was discovered. This effect appears for all analyzed circuits. We start with a simplest electronic circuit that has two parameters only $(N=2)$ and doesn't has any practical sense, but services well to understand the processes that occur in the design procedure. Then we analyze the $N$-dimensional problem, where $N$ has variation from 5 to 14 . All these examples demonstrate the additional acceleration effect that appears due to the different design trajectory behavior with the different control functions.

\subsection{Two-dimensional problem}

There is an analysis of a simplest electronic circuit with the topology, which is shown in Fig. 1.

We suppose that the element $r_{1}$ has a non-linear dependency in general case: $r=r_{10}+b_{n} \cdot V_{1}^{2}$. There are only two variable parameters in this circuit, the resistance $r_{2}$ and the voltage $V_{1}$. The element $r_{2}$ is supposed as an independent parameter $(K=1)$ and the voltage $V_{1}$ as a dependent parameter $(M=1)$. 


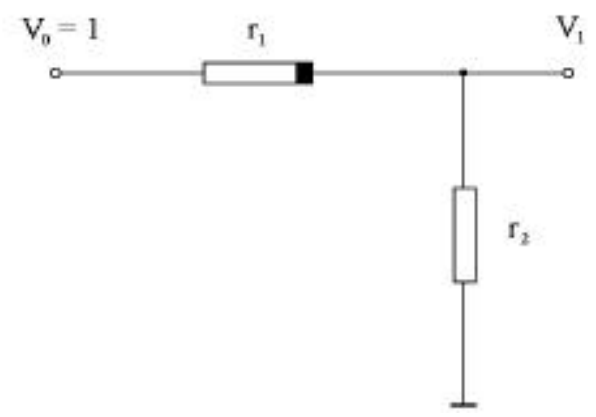

Figure 1. Topology of a simplest electronic circuit

Vector $X$ of the state variables has two components $X=\left(x_{1}, x_{2}\right)$ where $x_{1}^{2} \equiv r_{2}, x_{2} \equiv V_{1}$. The model of the system is given by: $x_{2}=\frac{x_{1}^{2}}{x_{1}^{2}+r_{10}+b_{n} x_{2}^{2}}$. This equation is transformed to the normal form as:

$$
g_{1}(X) \equiv\left(x_{1}^{2}+r_{10}+b_{n} x_{2}^{2}\right) x_{2}-x_{1}^{2}=0
$$

The objective function is defined by the formula $C(X)=\left(x_{2}-k_{V}\right)^{2}$, where $k_{V}$ has the fixed value. There is only one control function $u_{1}$ in this case because there is only one dependent parameter $x_{2}$. The design trajectory for this example is the curve in two-dimensional space, if the numerical design algorithm is applied. At the same time, the numerical analysis of this simple circuit doesn't have sense, because there is an analytic solution for this problem. We can obtain this solution by means of the Lagrange multipliers for example. However, we provide the numerical analysis of this circuit to reveal the essential features of the new additional design process acceleration effect. The main features of this analysis appear in all other examples too.

The optimization procedure and the electronic system model, in accordance with the new design methodology [3], are defined by the next two equations:

$$
\begin{aligned}
& x_{i}^{s+1}=x_{i}^{s}+t_{s} \cdot f_{i}(X, U), \quad i=1,2 \\
& \left(1-u_{1}\right) g_{1}(X)=0
\end{aligned}
$$

where $U$ is the vector of control variables, and the components of the movement directions $f_{i}(X, U)$ for the $i=1,2$ depend on the optimization method.
These functions, for the gradient method for example, are given by the formulas [1]:

$$
\begin{aligned}
& f_{1}(X, U)=-\frac{\delta}{\delta x_{1}} F(X, U) \\
& f_{2}(X, U)=-u_{1} \frac{\delta}{\delta x_{2}} F(X, U)+\frac{\left(1-u_{1}\right)}{t_{s}}\left[-x_{2}^{s}+\eta_{2}(X)\right]
\end{aligned}
$$

where $F(X, U)$ is the generalized objective function, $F(X, U)=C(X)+\frac{1}{\varepsilon} u_{1} g_{1}^{2}(X), \quad \eta_{2}(X)$ is the implicit function $\left(x_{2}^{s+1}=\eta_{2}(X)\right)$ and it gives the value of the parameter $x_{2}$ from the equation (3), and the operator $\frac{\delta}{\delta x_{i}}$ for $i=1,2$ means: $\frac{\delta}{\delta x_{1}} F=\frac{\partial F}{\partial x_{1}}+\frac{\partial F}{\partial x_{2}} \frac{\partial x_{2}}{\partial x_{1}}, \frac{\delta}{\delta x_{2}} F=\frac{\partial F}{\partial x_{2}}$.

The results of this circuit design for the nonlinearity parameter $b_{n}=1.0$ and for three different optimization methods, the gradient method, the Newton's method, and the Davidon-Fletcher-Powell method (DFP) are given in Table 1 for the traditional design strategy $\left(u_{1}=0\right)$ and for the modified traditional strategy $\left(u_{1}=1\right)$. The initial point of the

\begin{tabular}{|c|c|c|c|c|c|c|}
\hline \multirow[t]{2}{*}{ N Control fundions } & \multicolumn{2}{|c|}{ Gadent method } & Nenton & method & DP & mathod \\
\hline & $\begin{array}{l}\text { therations } \\
\text { number }\end{array}$ & $\begin{array}{l}\text { Totaldesign } \\
\text { time(sec) }\end{array}$ & $\begin{array}{l}\text { therations } \\
\text { number }\end{array}$ & $\begin{array}{l}\text { Totaldesign } \\
\text { time(sec) }\end{array}$ & $\begin{array}{l}\text { llerations } \\
\text { number }\end{array}$ & $\begin{array}{l}\text { Totaldesign } \\
\text { time }(\mathrm{sec})\end{array}$ \\
\hline 1 & 9 & 0.001018 & 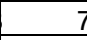 & 0.001653 & 7 & 0.00142 \\
\hline 2 & 72 & 0.006768 & 11 & 0.002931 & 12 & 2002 \\
\hline
\end{tabular}
vector $X$ for the system design is the next: $X_{i n}=(1,1)$.

Table 1. Complete set of design strategies for the initial

$$
\text { vector } X_{i n}=(1,1) \text {. }
$$

The traditional design strategy is the optimal one in this case and it cannot be improved when the initial vector $X_{\text {in }}$ is defined as $(1,1)$. The trajectories of the design process for this case are very simple to draw. We have a two-dimensional design phase space in this case. The trajectories which correspond to the gradient optimization method from the Table 1 for the initial vector $X_{\text {in }}$ with the components $(1,1)$ and for three different values of the non-linearity parameter $b_{n}\left(10^{-5}, 1.0,5.0\right)$ are presented in Fig. 2 (a), (b), (c). 


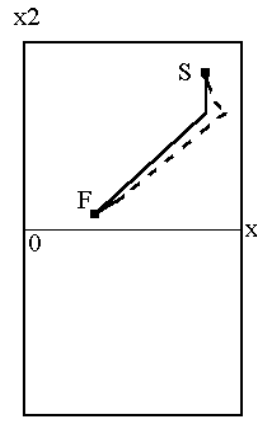

(a)

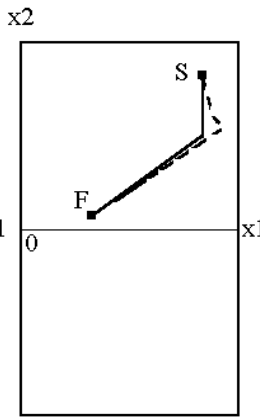

(b)

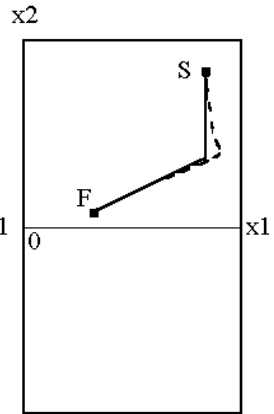

(c)
Figure 2. Trajectories for the traditional strategy (solid line) and for the modified traditional strategy (dash line) for $X_{\text {in }}=(1,1)$. a) $b_{n}=10^{-5}$; b) $b_{n}=1.0$; c) $b_{n}=5.0$.

Solid lines in this figure correspond to the traditional design strategy $\left(u_{1}=0\right)$; dash lines correspond to the modified traditional strategy $\left(u_{1}=1\right)$. The optimal trajectories coincide with trajectories of the traditional design strategy. Another trajectory behavior is observed when the initial value of the parameter $x_{2}$ is negative. The trajectories for the three above described situation are presented in Fig. 3 (a), (b), (c), for $X_{\text {in }}=(1,-1)$.

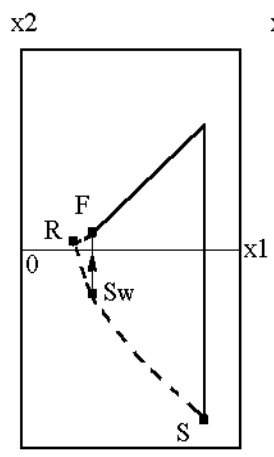

(a)

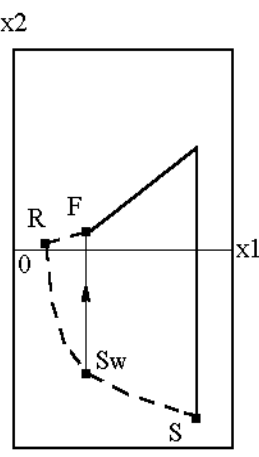

(b)

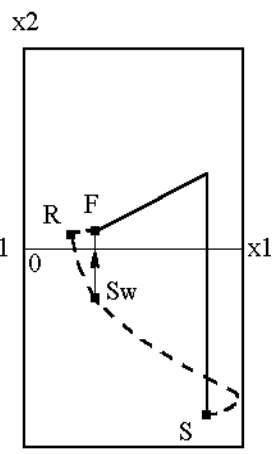

(c)
Figure 3. Trajectories for the traditional strategy (solid line) and for the modified traditional strategy (dash line) for $X_{i n}=(1,-1)$. a) $b_{n}=10^{-5}$; b) $b_{n}=1.0$; c) $b_{n}=5.0$.

The trajectories that correspond to the traditional design strategy practically do not have dependency from the initial value of the component $x_{2}$. There is an only jump in the start point $S$ to the principal part of the trajectory line from above (when $x_{2}=1$, Fig.2) or from below (when $x_{2}=-1$, Fig.3). Another situation is observed when the modified traditional strategy is used for $x_{2}=-1$. The first part of the trajectory lies in a physically unreal sub-space $\left(x_{2}<0\right)$ and the second part lies in a real sub-space $\left(x_{2}>0\right)$. Moreover, it is very important to note that the movement along the trajectory is very fast from the start point $S$ to the point $R$. On the other hand the movement is by far more slow from the point $R$ to the finish point $F$. It is very important that trajectories which correspond to the traditional and the modified traditional strategies draw to the finish point $F$ from the opposite directions. The unique possibility to accelerate the design process is created when the switching point of the control function $u_{1}$ lies in the point, which is the projection of the finish point $F$ to the modified traditional strategy trajectory, which lies in unreal sub-space. This is the point $S w$. The optimal trajectory has two parts in this case. The first part corresponds to the curve $S$ - $S w$. During the movement along this curve the control function $u_{1}$ is equal to 1 . The control function $u_{1}$ at the time moment, which corresponds to the point $\mathrm{Sw}$ changes the value to 0 . At this moment the jump is realized from the point $S w$ to the finish point $F$ or very near to the point $F$ (it depends on the calculate step). Therefore a great acceleration of the design process takes place. This acceleration effect is observed for all values of the non-linearity parameter $b_{n}$. The data, which correspond to the non-linearity parameter $b_{n}=1.0$, initial vector $X_{i n}=(1,-1)$ and three different optimization methods are given in Table 2 for the optimal design strategy.

Table 1. Complete set of design strategies for the initial vector $X_{\text {in }}=(1,-1)$.

\begin{tabular}{|l|l|r|r|r|l|}
\hline $\mathrm{N}$ & Method & $\begin{array}{l}\text { Optimal control } \\
\text { function u1 }\end{array}$ & $\begin{array}{l}\text { Iterations } \\
\text { number }\end{array}$ & $\begin{array}{l}\text { Switching } \\
\text { points }\end{array}$ & $\begin{array}{l}\text { Total } \\
\text { design } \\
\text { time (sec) }\end{array}$ \\
\hline 1 & Gradient method & $1 ; 0$ & 2 & 1 & 0.0002071 \\
\hline 2 & Newton method & $1 ; 0$ & 2 & 1 & 0.0005025 \\
\hline 3 & DFP method & $1 ; 0$ & 2 & 1 & 0.0004043 \\
\hline
\end{tabular}

The optimal trajectory has two parts for all optimization methods. The computer time gain of the optimal design strategies with respect to the traditional design strategy by the acceleration effect is equal to $4.91,3.29,3.53$ for the gradient method, Newton method and DFP method respectively. This effect is observed for more complicate examples too. However, in this case a trajectory line of the design process lies in $\mathrm{N}$-dimensional design space and we need to analyze different projections of $\mathrm{N}$ dimensional curves. 


\subsection{Five-dimensional problem}

The topology of the circuit is shown in Fig. 4.

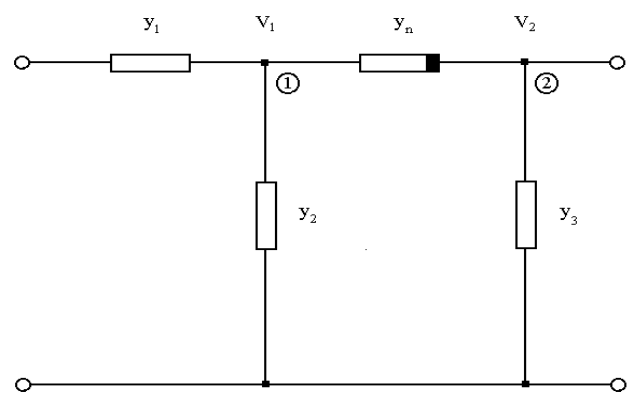

Figure 4. Circuit topology for $\mathrm{K}=3, \mathrm{M}=2$

This is a non-linear circuit that has three admittance $y_{1}, y_{2}, y_{3}$ as independent parameters, $(K=3)$ and two node voltages $V_{1}, V_{2}$ as dependent parameters, $(M=2)$. Non-linear element has dependency by the law: $y_{n}=a_{n}+b_{n} \cdot\left(V_{1}-V_{2}\right)^{2}$. The vector $X$ has five components $X=\left(x_{1}, x_{2}, x_{3}, x_{4}, x_{5}\right)$ where $x_{1}^{2} \equiv y_{1}$, $x_{2}^{2} \equiv y_{2}, x_{3}^{2} \equiv y_{3}, x_{4} \equiv V_{1}, x_{5} \equiv V_{2}$. The objective function $C(X)$ has been determined as the sum of the squared differences between beforehand-defined values and current values of the nodal voltages for two nodes with additional inequalities for some circuit elements. However, it can be noted that the additional acceleration effect appears for the different types of the objective function. The data of the complete set of design strategy with constant value of the control function vector $U$ and positive components of the initial vector $X_{\text {in }}$ are presented in Table 3 for three different optimization procedures.

Table 3. Complete set of design strategies for the initial vector $X_{\text {in }}=(1,1,1,1,1)$.

\begin{tabular}{|c|c|c|c|c|c|c|c|}
\hline \multirow[t]{2}{*}{$\mathrm{N}$} & \multirow{2}{*}{$\begin{array}{l}\text { Control functions } \\
\text { vector } \mathrm{U} \text { (u1, u2) }\end{array}$} & Gradient & method & \begin{tabular}{|l|l} 
Newton \\
\end{tabular} & method & DFP & method \\
\hline & & $\begin{array}{l}\text { lterations } \\
\text { number }\end{array}$ & $\begin{array}{l}\text { Total design } \\
\text { time (sec) }\end{array}$ & \begin{tabular}{|l} 
Iterations \\
number
\end{tabular} & $\begin{array}{l}\text { Total design } \\
\text { time (sec) }\end{array}$ & \begin{tabular}{|l|}
$\begin{array}{l}\text { Iterations } \\
\text { number }\end{array}$ \\
\end{tabular} & \begin{tabular}{|l} 
Total design \\
time $(\mathrm{sec})$
\end{tabular} \\
\hline 1 & $(00)$ & $\overline{16}$ & 0.0243 & 7 & 0.0396 & 8 & 0.0241 \\
\hline 2 & ( & 51 & 0.0238 & 9 & 0.0251 & 10 & 0.0107 \\
\hline 3 & $(1$ & 60 & 0.0448 & 8 & 0.0329 & 21 & 0.0331 \\
\hline 4 & (11) & 68 & 0.0217 & 11 & 0.0231 & 23 & 0.0198 \\
\hline
\end{tabular}

All these strategies are not time-optimal and the optimal design strategies for all optimization methods were found by means of the additional analysis. The results of this analysis are given in Table 4 for the non-linearity parameters $b_{n}=1.0$ and for two values of the initial vector $X_{i n}=(1,1,1,1,1)$ and $X_{i n}=(1,1,1,1,-1)$.
Table 4. Data of the optimal design strategies for two values of the initial vector

$$
X_{\text {in }}=(1,1,1,1,1), X_{\text {in }}=(1,1,1,1,-1) .
$$

\begin{tabular}{|l|l|l|l|l|l|l|}
\hline $\mathrm{N}$ & Method & $\begin{array}{l}\text { Initial co-ordinate } \\
\text { vector Xin }\end{array}$ & $\begin{array}{l}\text { Optimal control } \\
\text { functions vector } \\
\mathrm{U}(\mathrm{u} 1, \mathrm{u} 2)\end{array}$ & $\begin{array}{l}\text { Iterations } \\
\text { number }\end{array}$ & $\begin{array}{l}\text { Switching } \\
\text { points }\end{array}$ & $\begin{array}{l}\text { Total } \\
\text { design } \\
\text { time }(\mathrm{sec})\end{array}$ \\
\hline 1 & Gradient method & $(1,1,1,1,1)$ & $(10) ;(11)$ & 39 & 11 & 0.0141 \\
& $(1,1,1,1,-1)$ & $(11) ;(00) ;(11)$ & 16 & $2 ; 3$ & 0.0063 \\
\hline 2 & Newton method & $(1,1,1,1,1)$ & $(11) ;(10)$ & 7 & 3 & 0.0228 \\
& & $(1,1,1,1,-1)$ & $(10) ;(00) ;(01)$ & 5 & $1 ; 2$ & 0.0181 \\
\hline \multirow{2}{*}{3} & DFP method & $(1,1,1,1,1)$ & $(01) ;(11)$ & 10 & 9 & 0.0115 \\
& $(1,1,1,1,-1)$ & $(11) ;(01)$ & 7 & 2 & 0.0071 \\
\hline
\end{tabular}

These results correspond to the analysis of the previous section. The optimal control functions and the optimal behavior of the design trajectories were obtained on the basis of some approximate methods of the optimal control theory [4]-[10]. The computer time gain of the optimal design strategy with respect to the traditional design strategy is equal to 1.73 , 1.74, and 2.3 for the gradient method, Newton method and DFP method respectively and for the first value of the initial vector $X_{i n}$. An additional acceleration effect is displayed in case when the initial vector $X_{\text {in }}$ is equal to one of the two possible values: $(1,1,1,1,-1)$ or $(1,1,1,-1,-1)$. More effect is observed for the first value. This effect appears due to the trajectory jump, similar to the twodimensional problem of the previous section. However, in this case we have five-dimensional space problem and the trajectory behavior is more complicated. The computer time gain in this case is equal to $3.85,2.19$, and 3.41 for three above mentioned optimization methods. So, in this case we have an additional time gain of $123 \%, 26 \%$, and $48 \%$ for three different methods.

\subsection{N-dimensional problem}

In general case, we have $N$-dimensional design problem. However, all specific features of the additional design acceleration, as a necessary trajectory jump, and a time gain are revealed again. The potential computer time gain of the optimum design strategy without and with an additional acceleration as the function of the dependent parameters' number $M$ is presented in Fig. 5 (a), (b) for three different optimization procedures.

Fig. 5 (a) corresponds to the time gain without an additional acceleration effect when the initial value of the state variables are positive and Fig. 5 (b) corresponds to the time gain with an additional acceleration effect when the initial value of some state variables are negative. 


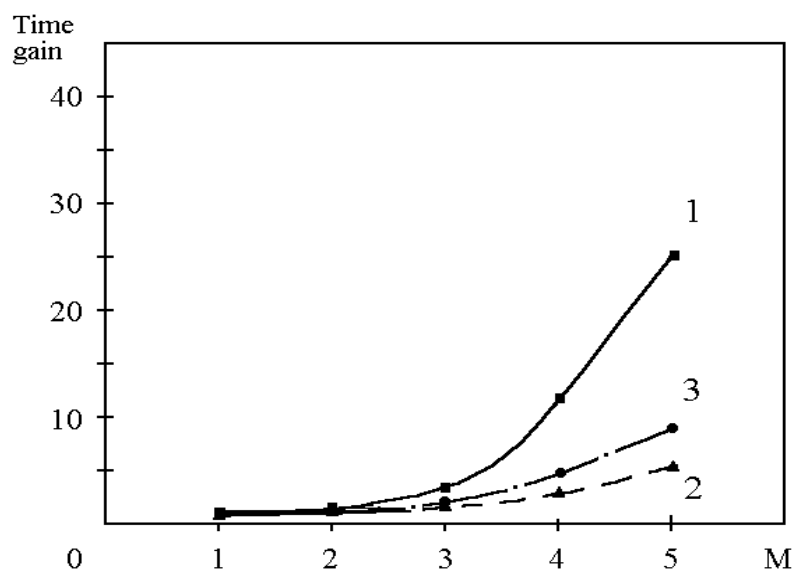

(a)

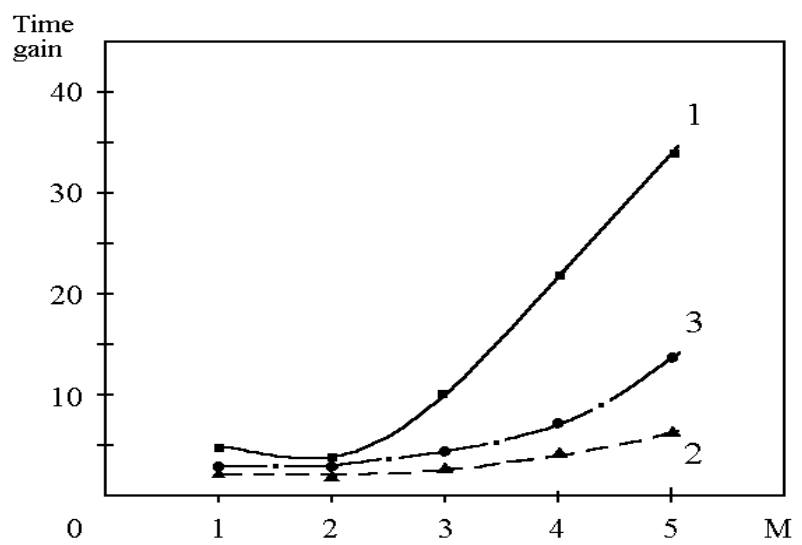

(b)

Figure 5. Optimal strategy potential computer time gain. 1-Gradient method, 2-Newton method, 3-DFP method.

(a) without an additional acceleration effect; (b) with an additional acceleration effect.

The circuit topology for the different node number $M$ has been taken from the paper [3]. The comparison of the curves of the figures 5 (a) and 5 (b) demonstrates that the additional acceleration effect is displayed for all analyzed examples and gives an additional time gain from $20 \%$ to $180 \%$ depending on the problem dimension and optimization method.

The active circuit analysis gives similar results. In Fig. 6 there is a circuit of the amplifier that consists of three transistor cells.

There are three-node circuit for one transistor cell, the five-node circuit for the two transistor cells and the seven-node circuit for the three transistor cells. The potential computer time gain of the optimum design strategy without and with an additional acceleration as the function of the transistor cell number $N_{T R}$ is presented in Fig. 7 (a), (b) for two

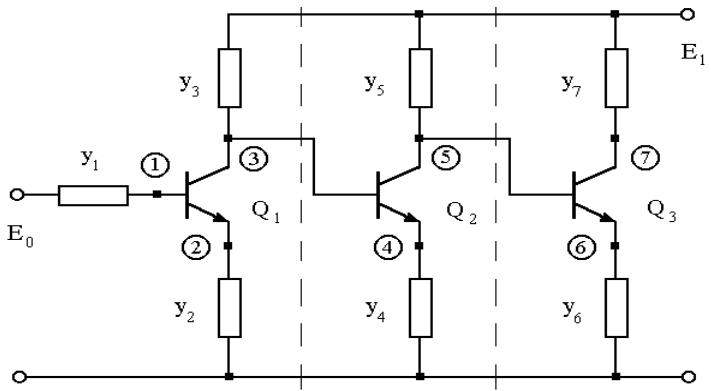

Figure 6. Circuit topology for three-transistor cell amplifier.

different optimization procedures (gradient method and DFP method). The additional acceleration effect is observed for the active circuit too, when some components of the initial vector $X_{i n}$ are negative.

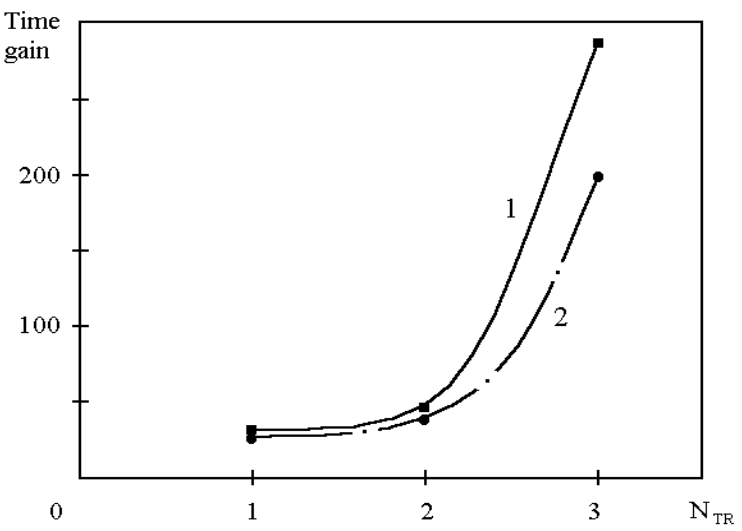

(a)

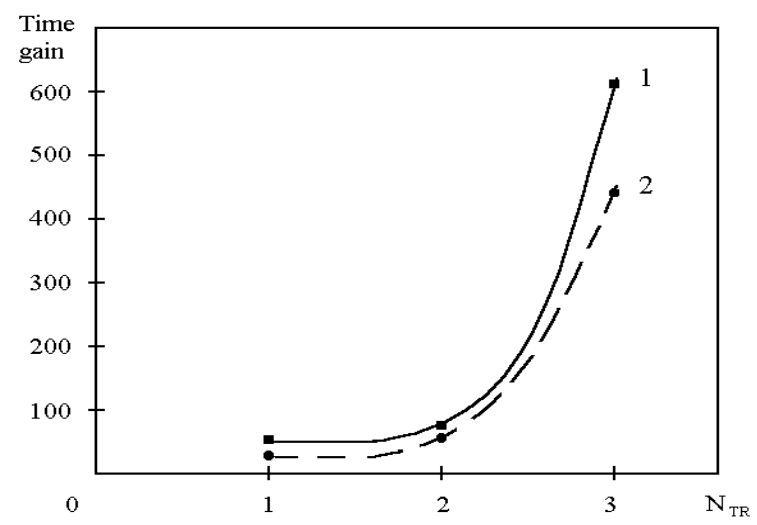

(b)

Figure 7. Optimal strategy potential computer time gain. 1-Gradient method, 2-DFP method.

(a) without an additional acceleration effect; (b) with an additional acceleration effect. 
However, in this case the analysis is more complicated because the trajectory design line not always exists due to the specific current dependency of the transistor junctions. The additional time gain due to the acceleration effect is changed from $30 \%$ to $125 \%$ depending on the node number and the optimization method. The trajectory behavior near the finish point has a grate influence to the acceleration effect quantitative value. The complex behavior of the trajectories can complicate the acceleration effect achievement because there are more than one jump required in this case. The total computer time gain of the optimal strategy for the last example (three transistor cells circuit with 7 nodes and 14 variables) due to the acceleration effect is equal to 620 for the gradient optimization method and 477 for the DFP method.

This value of the computer time gain shows a great perspective of further research in this direction. Now it is clear that the start point of the optimal design process must be elected with at least one negative coordinate and the first part of the optimal design trajectory lies in unreal state space. The other part of the optimal design strategy consists of one or several jumps with the special adjust trajectories of the different admissible strategies.

The additional acceleration effect, which is discussed here, serves as an excellent example of a new qualitative result, which was obtained by the generalized system design methodology. It is clear that all these advantages of the new approach are realized when the time-optimal algorithm is constructed. One of the main problems on this way is the definition of the specific characteristics and special features of the optimal or quasi-optimal design algorithm. The results obtained here serve as the first step for the optimal design algorithm characteristic determined in particularly for the initial point optimal selection and for the preliminary definition of the optimal trajectory and control function structure.

\section{Conclusions}

The analysis of the different design strategies has been done on the basis of before elaborated new system design methodology. The design trajectory behavior was analyzed for the different initial value of the state variables. The additional acceleration effect of the system design process was discovered by means of the variation of the initial value of the state variables and the special control functions. This effect exists owing to the very different behavior of the design trajectories that have various control functions and different start points of the design space. This new effect reduces the total computer time additionally and gives the perspective to accelerate more the system design process. On the other hand, the obtained results give the useful information about the initial point selection for the optimal design process and about the structure of the optimal or quasi-optimal design trajectory.

\section{References:}

[1] A. Zemliak, One Approach to Analog System Design Problem Formulation, Proc. of the 2001 IEEE Int. Symp. on Quality Electronic Design, San Jose, USA, March, 2001, pp. 273-278.

[2] A. Zemliak, System Design Problem Formulation by Control Theory, Proc. of the IEEE Int. Symp. Circuits Systems, Sydney, Australia, May, 2001,Vol. 5, pp. 5-8.

[3] A. Zemliak, Analog System Design Problem Formulation by Optimum Control Theory, IEICE Trans. on Fundamentals of Electronics, Comunications and Computer Sciencies, Vol. E84-A, No. 8, 2001, pp. 2029-2041.

[4] J.B. Rosen, Iterative Solution of Nonlinear Optimal Control Problems, J. SIAM, Control Series A, pp. 223-244, 1966.

[5] Tabak D., and B.C.Kuo, Applications of Mathematical Programming in the Design of Optimal Control Systems, Int. Journal of Control, Vol. 10, No. 5, 1969, pp. 548-552.

[6] I.A.Krylov, and F.L. Chernousko, Consecutive Approximation Algorithm for Optimal Control Problems, J. of Numer. Math. and Math. Pfysics, vol. 12, no 1, pp. 14-34, 1972.

[7] R.P. Fedorenko, Approximate Solution of Optimal Control Problems, Nauka, Moscow, 1978.

[8] V.F. Krotov, Global Methods in Optimal Control Theory, Marcel Dekker, Inc., N.Y., 1996.

[9] Sepulchre R., M. Jankovic, and P.V. Kokotovic, Constructive Nonlinear Control, SpringerVerlag, N.Y., 1997.

[10] R. Pytlak, Numerical Methods for Optimal Control Problems with State Constraints, Springer-Verlag, Berlin, 1999. 